\title{
Aspects of Modeling Piezoelectric Active Thin-walled Structures
}

\author{
Dragan MARINKOVIĆ, ${ }^{1,2, *}$ HeINZ KÖPPE ${ }^{3}$ AND UlRich GABBERT ${ }^{3}$ \\ ${ }^{1}$ Technical University of Berlin, Institute of Mechanics, Strasse des 17. Juni, D-10623 Berlin, Germany \\ ${ }^{2}$ Mechanical Faculty, University of Niš, Aleksandra Medvedeva 14, Niš, Serbia \\ ${ }^{3}$ Otto-von-Guericke-University Magdeburg, Institute of Mechanics, Universitätsplatz 2 \\ D-39106 Magdeburg, Germany
}

\begin{abstract}
The objective of this article is to reconsider some important aspects of modeling piezoelectric active thin-walled structures. Hence, it is dealt here with thin-walled laminated structures involving piezoelectric patches. A recently developed shell type finite element is used for the purpose. The first aspect is adequate modeling of electric field within the piezoelectric patches polarized in the thickness direction. The influence of higher order functions for the electric field on the accuracy of the model is discussed. The second aspect is related to modeling geometrical non-linearities in the behavior of the considered structures and their significance on the accuracy of the predicted behavior. Both aspects are considered with respect to static and dynamic cases.
\end{abstract}

Key Words: piezoelectric composites, electric field modeling, geometrical non-linearities.

\section{INTRODUCTION}

G ENERALLY speaking, an active structure is a Istructure that includes bonded or embedded multi-functional material-based active elements - sensors and/or actuators, the purpose of which is to monitor the state of the structure and to produce desired excitations affecting the structure, respectively. In further step, sensors are coupled with actuators by means of a controller, which enables the structure to respond to external stimuli to compensate for undesired behavior or to produce desired one. Such a structure is denoted as adaptive.

The interest for the piezoelectric active thin-walled structures have significantly increased over the last two decades. A number of researchers have been attracted to the field, which is due to the potential and already used benefits active structures offer over passive ones. A large group of research projects is dedicated to new industrial applications of active structures or the improvement of already existing applications. The other group deals generally with the problem of modeling active structures. The main aim here is to provide adequate models, modeling tools, and to give new insights into the issue. Namely, in any analysis scenario, it is necessary at first to decide the significant aspects of the physical processes to be modeled. Hereafter, model building

*Author to whom correspondence should be addressed. E-mail: Dragan.Marinkovic@TU-Berlin.de

Figure 4 appears in color online: http://jim.sagepub.com explores alternative solutions with the aim of achieving a satisfying compromise between the model complexity and the accuracy of the predicted behavior of the physical system - the two objectives that are definitely not easy to conciliate. The aspects of modeling piezoelectric active structures that are considered in the article are investigated by means of finite element (FE) method with a recently developed shell type FE used for the purpose.

The first aspect to be investigated is modeling the electric field quantities in the piezoelectric active elements. Within the last few years a number of papers have been published, in which a quadratic distribution of the electric potential, and correspondingly, a linear one for the electric field through the piezolayer thickness was accounted for (e.g., Yang et al., 2004; Butz and Klinkel, 2005; Cotoni, 2006). The mentioned papers also give significance to this issue, while, on the other hand, a great number of previously developed formulations take a priori a linear and constant distribution, respectively, and also yield results of good accuracy (e.g., Gabbert et al., 1998; Piefort, 2001). This gave impetus to investigation on this matter. The investigation presented in this article is actually a sequel to a similar consideration presented by the authors in Marinković et al. (2007).

The second aspect to be investigated is related to geometrical non-linearities in the behavior of active thinwalled structures. A relatively small body of literature is available on this matter and that was the main motivation to address this aspect here. Recently some researchers have given attention to this issue (e.g., Rabinovitch, 2005; 
Kulkarni and Bajoria, 2007; Marinković et al., 2008). If a model-based control is to be developed in order to render active structure an adaptive one, it is of crucial importance to have a model capable of predicting the actual behavior with a satisfying accuracy. Such a level of accuracy might require consideration of geometrically non-linear effects. The intention of the authors is to present briefly the developed geometrically non-linear formulation and to investigate this aspect on a relatively simple example involving both static and transient behavior of a structure.

\section{SHELL ELEMENT FOR THIN-WALLED ACTIVE STRUCTURES}

Only the most important features of the developed element will be given here, since a thorough description of the element formulation is already available in Marinković et al. (2006) and Marinković (2007). The effective modeling and simulation of the considered structures are driven by the recognition that the nature of their general behavior allows the condensation of the complex 3D field to the essential ingredients of the structural response described by a 2D approach. Of all the FEs developed for plate and shell structures, the degenerated shell element family is widely considered to be one of the most efficient and economic. The choice of the authors goes in the same direction.

The developed element is a 9-node shell element and is applicable over a wide range of thickness and curvatures. The kinematical assumptions made in the formulation of the element are fully compatible with the Mindlin-Reissner kinematical assumptions (first-order shear deformation theory). Thus, the transverse shear strains and stresses are included in the formulation and, consequently, the element can be used for modeling thin as well as moderately thick structures.

In the formulation of the element three different coordinate systems have been used: global $(x, y, z)$, local $\left(x^{\prime}, y^{\prime}, z^{\prime}\right)$, and natural (r, s, t) (Figure 1). The global c.s. is a Cartesian c.s. with a fixed position in space. The behavior of the structure is described with respect to this c.s. The natural c.s. has dimensionless coordinates taking values between -1 and +1 , where $r$ and $s$ are the

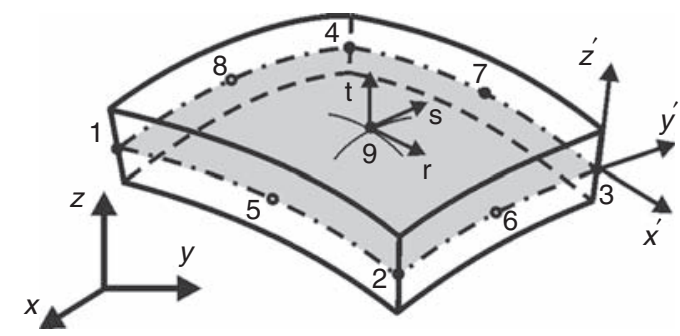

Figure 1. Coordinate systems of the ACShell9 element. curvilinear coordinates in the reference surface, while $\mathrm{t}$ is linear in the thickness direction. The local c.s. is defined at each point of the structure reference surface so as to have one of its axis (the $z^{\prime}$-axis) perpendicular to the reference surface. This c.s. provides an effective way of handling directionally dependent material properties, e.g., when the passive material of the structure comprises fiber-reinforced composite layers. The local c.s. is also used in the definition of the element geometry, displacement, strain and stress field, and it comes very handy for the description of the piezoelectric coupling. Namely, the coupling based on the ' $\mathrm{e}_{31}$ ' effect is achieved between the in-plane strain components and electric voltage acting in the thickness direction. Both quantities are effectively described in the local coordinate system regardless of the complexity of the structure's configuration (e.g., curvature) or the change of configuration throughout the analysis.

The developed shell element has six mechanical degrees of freedom (three translations and three rotations) at each of the nine nodes, and additionally, as many electrical degrees of freedom as there are embedded/bonded piezoelectric layers. The formulation assumes that the piezoelectric layers are polarized in the thickness direction only and that the differences of electric potentials between the lower and upper face of piezolayers are constant over the surface of the element. Those are actually the electrical degrees of freedom. The integration of the element vectors and matrices in the thickness direction is performed analytically, while the in-plane integration is performed numerically.

The element is capable of modeling linear and quadratic distribution of electric voltage across the thickness of piezoelectric layers. The user is given the choice which one to use. The element also supports geometrically non-linear analysis based on the updated Lagrangian formulation. The natural strains and Cauchy stresses are determined with respect to the local coordinate system.

The element is named ACShell9 (9-node active composite shell).

\section{MODELING OF ELECTRIC FIELD QUANTITIES IN PIEZOPATCHES}

As it is aforementioned, the authors have already made an investigation into the matter of accurate modeling of electric field quantities within the piezoelectric layers (Marinković et al., 2007). In the investigation, this aspect was examined considering only static cases. The objective here is to extend the investigation by considering additionally the influence of modeling piezolayers' electric field quantities on the dynamical behavior of structure. For the sake of completeness, the most 
important features from the preceding investigation as well as a small set of static cases will also be given here.

The form of the linear piezoelectric constitutive equations depends on the choice of independent variables (Ikeda, 1996). Choosing the mechanical strain and electric field as independent variables they read:

$$
\begin{aligned}
\{\sigma\} & =\left[\mathrm{c}^{\mathrm{E}}\right]\{\varepsilon\}-[\mathrm{e}]^{\mathrm{T}}\{\mathrm{E}\} \\
\{\mathrm{D}\} & =[\mathrm{e}]\{\varepsilon\}+\left[\mathrm{d}^{\varepsilon}\right]\{\mathrm{E}\}
\end{aligned}
$$

where $\{\sigma\}$ and $\{\varepsilon\}$ represent the mechanical stress and strain in the vector notation, respectively, $\{\mathrm{D}\}$ and $\{\mathrm{E}\}$ are the electric displacement and the electric field vector, respectively, $\left[\mathrm{c}^{\mathrm{E}}\right]$ is the Hooke's matrix at constant electric field, the matrix [e] comprises the piezoelectric coupling constants, and $\left[\mathrm{d}^{\varepsilon}\right]$ is the matrix of dielectric (permittivity) constants at constant strain.

It is dealt here with thin piezoelectric patches polarized in the thickness direction and with the electric field applied in the thickness direction (Figure 2). Hence, the quantities are written here with omitted indices and having in mind that they are always related to the thickness direction of the patch, which is at the same time the thickness direction of the structure. The electric potential, $\Phi$, and electric field, E, are related in the well-known way:

$$
\mathrm{E}=-\frac{\partial \Phi}{\partial \mathrm{z}_{\mathrm{P}}^{\prime}}
$$

where $z_{p}^{\prime}$ denotes the local thickness coordinate taking the zero value at the mid-surface of the piezopatch. Most of authors approach this issue by assuming the electric potential to be a linear function in $\mathrm{z}_{\mathrm{p}}^{\prime}$ and, correspondingly, the electric field to be constant:

$$
\mathrm{E}\left(\mathrm{z}_{\mathrm{P}}^{\prime}\right)=-\frac{\Delta \Phi}{\mathrm{h}_{\mathrm{P}}}=\text { const.; } \quad \Phi\left(\mathrm{z}_{\mathrm{P}}^{\prime}\right)=\Delta \Phi\left(\frac{\mathrm{z}_{\mathrm{P}}^{\prime}}{\mathrm{h}_{\mathrm{P}}}+\frac{1}{2}\right)
$$

with $\Delta \Phi$ (denoting the difference of electric potentials between the upper and lower faces of the piezopatch and $h_{P}$ is the thickness of the patch. This assumption is correct for patches exhibiting purely membrane behavior. Nevertheless, the function of active components attached to thin-walled structures is mainly related to bending behavior of the structures. In that case, it can be demonstrated that the consistent functions (i.e. satisfying the Gauss law) for the electric potential and

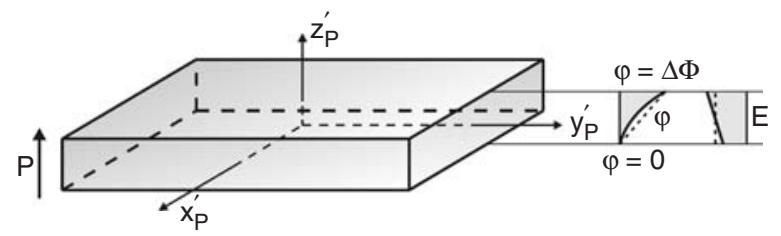

Figure 2. Piezopatch - electric field and electric potential. electric field are strongly related to the theory used to describe the structure's kinematics.

The piezoelectric materials belong to dielectrics. Since there are no free charges in dielectrics, the Gauss law reads:

$$
\frac{\partial \mathrm{D}}{\partial \mathrm{Z}_{\mathrm{P}}^{\prime}}=0
$$

Now, within a first-order theory for the kinematics of thin-walled structures, the in-plane displacements are assumed as linear in $\mathrm{z}_{\mathrm{p}}^{\prime}$, while the transverse deflection is assumed constant across the thickness. Correspondingly, the in-plane strains are linear in $z_{p}^{\prime}$ and they consist of purely membrane strains (constant term) and flexural strains related to the curvature of deformation $\kappa$ (linear term). Starting from the set of Equations (1) and (2), using the Gauss law and the aforementioned description of kinematics, a quadratic function in $z_{p}^{\prime}$ for the electric potential of piezopatch can be derived, as it is done in Marinković et al. (2007). Then it is obvious from (3) that the electric field is a linear function in $z_{p}^{\prime}$.

The same conclusion may be deduced in a more intuitive manner observing another set of piezoelectric constitutive equations that corresponds to the choice of mechanical strain and electric displacement as independent variables:

$$
\begin{aligned}
& \{\sigma\}=\left[\mathrm{c}^{\mathrm{D}}\right]\{\varepsilon\}-[\mathrm{h}]^{\mathrm{T}}\{\mathrm{E}\} \\
& \{\mathrm{E}\}=-[\mathrm{h}]\{\varepsilon\}+\left[\mathrm{b}^{\varepsilon}\right]\{\mathrm{D}\}
\end{aligned}
$$

with $[\mathrm{h}]$ comprising coupling constants and $\left[\mathrm{b}^{\varepsilon}\right]$ inpermittivity constants.

It is already elaborated that the Gauss law yields a constant value of dielectric displacement over the thickness of piezopatch. Hence, from (7) it is obvious that the function describing the electric field has to be of the same order in the thickness coordinate as the corresponding function for the mechanical strain, i.e., it depends on the theory chosen to describe the kinematics of the structure and is actually of the same order as the theory itself.

The electric potential and electric field functions consistent with the first-order shear deformation theory (first-order theory with shear deformation included) are derived in Marinković et al. (2007) and can be given here in a somewhat simplified form:

$$
\begin{aligned}
\Phi\left(\mathrm{z}_{\mathrm{P}}^{\prime}, \kappa\right)= & \frac{1}{2} \mathrm{k}_{\varphi}\left(\kappa, \mathrm{e}_{31}, \mathrm{~d}_{33}\right)\left(\mathrm{z}_{\mathrm{P}}^{\prime 2}-\left(\frac{\mathrm{h}_{\mathrm{p}}}{2}\right)^{2}\right) \\
& +\Delta \Phi\left(\frac{\mathrm{z}_{\mathrm{P}}^{\prime}}{\mathrm{h}_{\mathrm{p}}}+\frac{1}{2}\right) \\
\mathrm{E}\left(\mathrm{z}_{\mathrm{P}}^{\prime}, \kappa\right)= & -\frac{\partial \Phi\left(\mathrm{z}_{\mathrm{P}}^{\prime}, \kappa\right)}{\partial \mathrm{z}_{\mathrm{P}}^{\prime}}=-\mathrm{k}_{\varphi}\left(\kappa, \mathrm{e}_{31}, \mathrm{~d}_{33}\right) \mathrm{z}_{\mathrm{P}}^{\prime}-\frac{\Delta \Phi}{\mathrm{h}_{\mathrm{p}}}
\end{aligned}
$$

where $\mathrm{k}_{\varphi}$ is a coefficient depending on the piezoelectric constant $\mathrm{e}_{31}$, dielectric constant $\mathrm{d}_{33}$, and curvature 
of deformation $\kappa$. The information that should be extracted from (8) and (9) is that the highest order terms (quadratic for electric potential and linear for electric field) are related to the curvature of deformation, while the remaining of the both functions is the same as the functions used in typical approximations (4).

One may start from the Hamiton's principle in order to develop the FE equations for the piezoelectric continuum. The question that arises now is what difference the derived higher order functions make in the model. Following the formalism of developing the FE equations, the outcome of the electric field dependency on the curvature of the piezopatch deformation is an additional, purely mechanical stiffness in comparison with the model based on approximate functions (4) (Marinković et al., 2007). This additional stiffness can be given here in the following form:

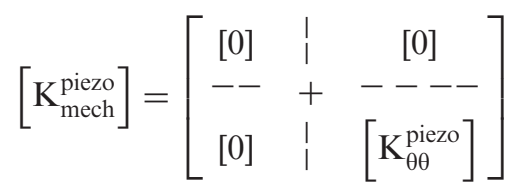

with

$$
\left[\mathrm{K}_{\theta \theta}^{\text {piezo }}\right]=\frac{2}{3}\left(\sum_{\mathrm{k}=1}^{\mathrm{N}_{\mathrm{pe}}} \mathrm{c}_{\mathrm{tg}_{\mathrm{k}}} \mathrm{c}_{\mathrm{pm}}\right) \int_{\mathrm{AP}_{\mathrm{P}}}\left[\mathrm{B}_{\mathrm{R} 1 \mathrm{f}}\right]^{\mathrm{T}}\left[\mathrm{B}_{\mathrm{P}}\right]\left[\mathrm{B}_{\mathrm{R} 1 \mathrm{f}}\right] \mathrm{dA}
$$

where $\mathrm{N}_{\mathrm{pe}}$ is the number of piezoelectric layers across the thickness, $A_{P}$ the surface covered by piezolayers, $c_{t_{k}}$ and $\mathrm{c}_{\mathrm{pm}_{\mathrm{k}}}$ are the thickness geometrical factor and piezoelectric material factor of the k-th piezolayer, respectively:

$$
\begin{aligned}
\mathrm{c}_{\mathrm{tg}_{\mathrm{k}}} & =\left(\frac{\mathrm{h}_{\mathrm{p}_{\mathrm{k}}}}{\mathrm{h}}\right)^{3} \\
\mathrm{c}_{\mathrm{pm}_{\mathrm{k}}} & =\left(\frac{\mathrm{e}_{31}^{2}}{\mathrm{~d}_{33}}\right)_{\mathrm{k}}
\end{aligned}
$$

$h$ is the overall thickness of the laminate, $\left[\mathrm{B}_{\mathrm{R} 1 \mathrm{f}}\right]$ is the part of the strain-displacement matrix defining flexural and torsional strains, and $\left[\mathrm{B}_{\mathrm{P}}\right]$ is a Boolean matrix which simply excludes the torsional strains from (11).

The additional stiffness is related to the rotational degrees of freedom only and is exhibited in bending dominated behavior. The more important question is if the difference in the results from the two models is pronounced enough to make the additional stiffness a necessary part of the model. In order to provide the answer, it would be worthwhile to give briefly the parameters, which determine the significance of the additional stiffness and which are identified from (11):

(1) The thickness geometrical factor $c_{t g}$ : The additional stiffness depends on the third power of the piezolayer thickness to the overall thickness ratio (see (12)).

(2) The piezoelectric material factor $c_{p m}$ : Larger values of the piezoelectric coupling constant increase the additional stiffness, while the dielectric constant has the opposite influence (see (13)).

(3) The surface covered by the piezolayers: The integration in (11) runs only over the part of the structure surface covered by the piezopatches.

(4) The 'genuine' mechanical stiffness of the material: If the bending stiffness of both passive and active material originating from their mechanical properties is much greater that the additional stiffness, then the influence of the latter will be practically unnoticeable.

\section{Static Examples}

The set of static examples given below is similar to examples from the previous investigation (Marinkovic et al., 2007). The examples are therefore not discussed in details. Actually, the results contain only the relative difference in displacements $(\Delta)$ obtained with different electric field formulations, as this is the focus here. The so obtained results are used to assess the significance of the aforementioned stiffening effect. The considered examples and the results are given tabularly starting from the well-known academic case of bimorph beam, which consists of two piezolayers only, then an active beam with a passive layer and finally a case of active plate (Table 1). For the sake of reproducibility of the examples, the material parameters as well as the dimensions are available in Table 1.

In all the considered cases the active layers have symmetric position with respect to the thickness of the structure and they act as actuators. They are oppositely polarized and supplied with the same electric voltage, which results in bending moments uniformly distributed over the edges of the surface covered by piezopatches. This results in bending-dominated behavior of the structure, which is a precondition for investigating the aspect at hand.

What should be observed here is the change of the above listed parameters influencing the significance of the investigated aspect. The thickness geometrical factor has obviously the highest possible value in the case of bimorph beam and is reduced going toward the active plate. The piezoelectric material factor is an important issue only in the first example. In this example, the whole structure is made of active, piezoelectric 
Table 1. Static examples for the investigation of additional stiffening effect.

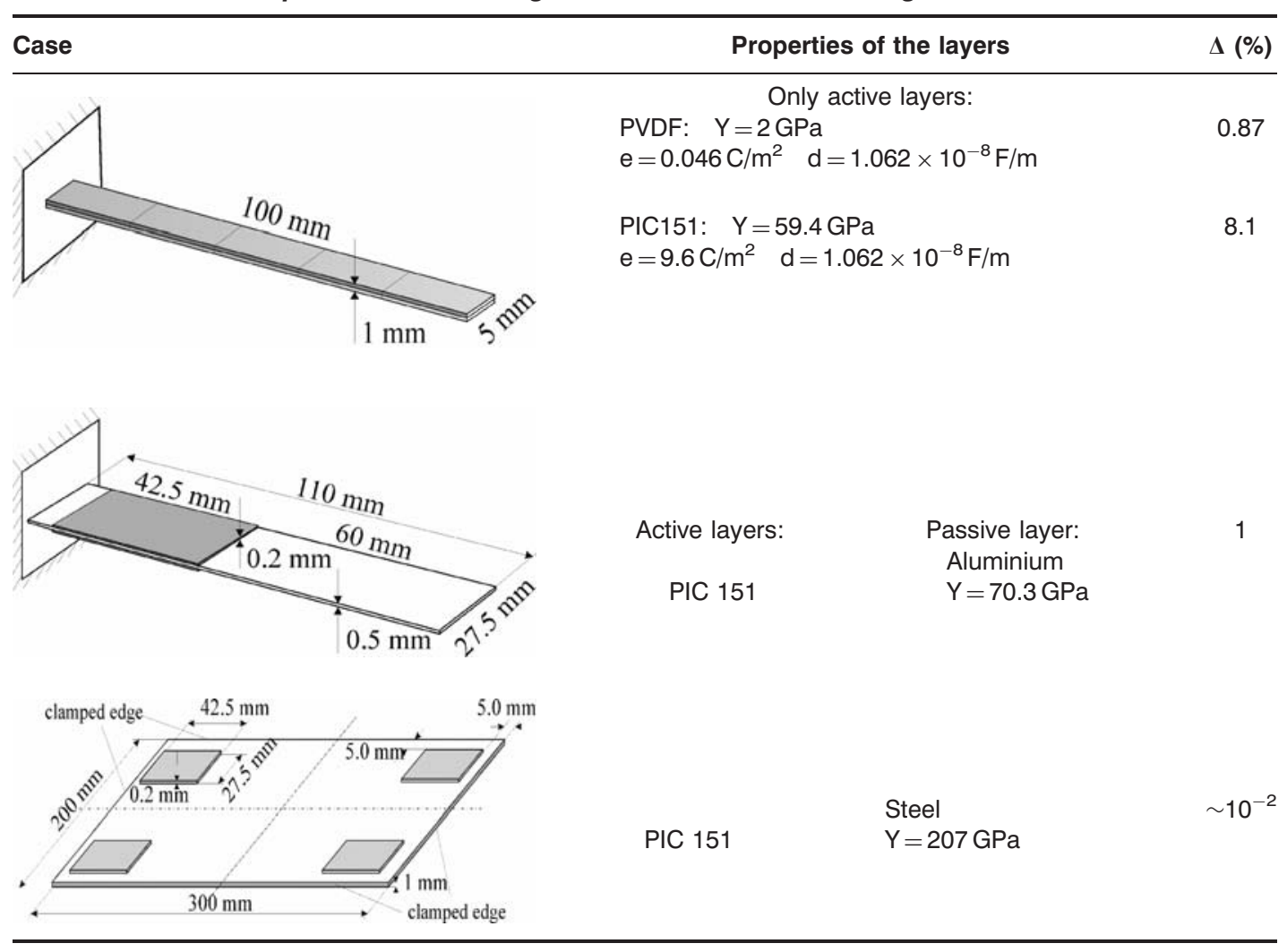

material only, and the beam is considered once to be made of polyvinylidene fluoride (PVDF) and then again to be made of piezoelectric ceramic PIC151. Although in this case all the parameters are set so as to yield the highest possible influence of the stiffening effect, when the structure is made of PVDF the significance of the effect is rather small. This is due to the very small values of the piezoelectric coupling constant. Hence, in further examples only PIC151 is considered as an active material, which means that the piezoelectric material factor remains unchanged. Regarding the surface covered by piezoelectric elements, obviously in the first example it is $100 \%$ of the structure. In the second example, $38.6 \%$ of the structure is covered by piezopatches, and in the third case, it is $7.8 \%$ of the structure. Finally, the genuine mechanical stiffness of the material increases going from the first toward the last example in the first example the considered material is active with no passive materials included, in the second example the passive material is aluminium and in the third it is steel. Also, the thickness of the passive materials increases throughout the examples.

The only remarkable difference between the results with the higher order functions for the electric quantities accounted for and the results obtained with typical approximate functions, is present in the very first example of the bimorph beam made of PIC151 - it is $8.1 \%$. Indeed, all the parameters in that case are chosen so as to yield the highest influence of the stiffening effect. In all other cases the difference is rather small and especially in the last considered case (active plate), where it is completely negligible. On the other hand, it should be noticed that the active plate example mostly resembles typical commercial application of piezoelectric active components - thin piezoelectric patches of rather small surface compared to the surface of the host structure.

As it was already concluded in Marinković et al. (2007), the considered examples point out that for typical applications of piezoelectric active elements on thin-walled structures (small thin patches attached to the structure's passive material), the usual approximations given here in (4) yield quite satisfactory results. In the following it should be considered if the same is valid for dynamic behavior of the structures.

\section{Dynamic Example}

A great part of dealing with active structures involves dynamics, such as vibration suppression, noise attenuation, fatigue damage protection, etc. This points out the necessity for investigation of the influence of considered stiffening effect on the dynamic behavior of piezoelectric structures. The following investigation is supposed to reveal how the eigenfrequencies of an active rectangular plate structure are affected by the effect.

A steel plate $\left(\mathrm{Y}=2.1 \times 10^{5} \mathrm{~N} / \mathrm{mm}^{2}, \quad \nu=0.3, \rho=\right.$ $7800 \mathrm{~kg} / \mathrm{m}^{3}$ ) with the in-plane dimensions $100 \times 50 \mathrm{~mm}^{2}$ 
and the thickness of $0.5 \mathrm{~mm}$, the upper surface of which is entirely covered with a $0.25 \mathrm{~mm}$ layer of piezoceramic (PIC151, $\rho=7800 \mathrm{~kg} / \mathrm{m}^{3}$ ) is considered. The plate is simply supported on two opposite longer sides. The steel plate is grounded and acts as an electrode. Additionally, 10 identical electrodes, each covering the whole width of the plate, are regularly distributed along the length of the plate (Figure 3). At first, the modal analysis of the plate is performed with the stiffening effect neglected for the piezolayer under all 10 electrodes, i.e., with the approximate functions (4) for the electric field and potential. In the next steps, the effect is taken into account gradually always for one more electrode starting from one of the free sides of the plate and going toward the opposite free side, thus gradually increasing the influence of the third parameter (surface covered by active elements). This may be understood as if the active layer was passive in the beginning with exactly the same mechanical and physical properties (but with no piezoelectric coupling), and afterwards, in each step this passive layer is exchanged for the original active layer (with piezoelectric coupling) under a new electrode (thus including the stiffening effect under the electrode).

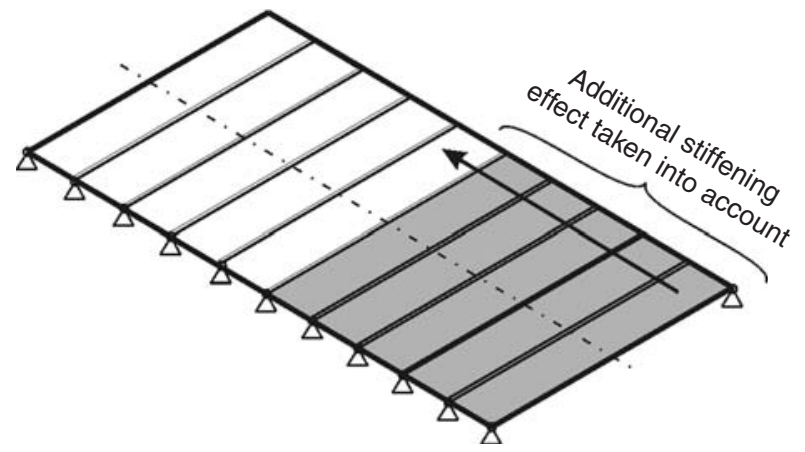

Figure 3. Model of active plate simply supported on two opposite sides.
The modal analysis is performed for each step and the change of the eigenfrequencies is observed.

The diagram in Figure 4 shows the relative increase of the eigenfrequencies for different modes. The reference eigenfrequency for each mode is the one obtained with the stiffening effect neglected for all 10 electrodes. It is easy to recognize that the eigenfrequencies continuously grow with the effect accounted for under each new electrode. Furthermore, as expected, the increase is quite small for the electrodes in the vicinity of the mode nodes, and oppositely, the fastest increase is noticeable for the electrodes around the mode antinodes (the points with maximal amplitudes). An approximately linear increase can be noticed for the bending modes along the width (the first and the fifth mode), which is due to the fact that each electrode covers the whole width and they are added in the direction of the length. In this case each new electrode results in approximately the same increase of the additional mechanical stiffness of the structure and, consequently, the same increase of the eigenfrequency of the mentioned modes.

Nevertheless, it may be noticed that the maximal change in eigenfrequencies (i.e., for $100 \%$ of the structure surface covered by active elements) is fairly below $1 \%$, which contributes to the conclusion given for static cases.

\section{GEOMETRICALLY NON-LINEAR EFFECTS IN BEHAVIOR OF THIN-WALLED ACTIVE STRUCTURES}

The prediction of the linearity of the system response rests on assumptions, which imply that the displacements of the FE assemblage are quite small with respect to the dimensions of the modeled structure and that the material exhibits a linear elastic behavior. An additional assumption says that the boundary conditions remain

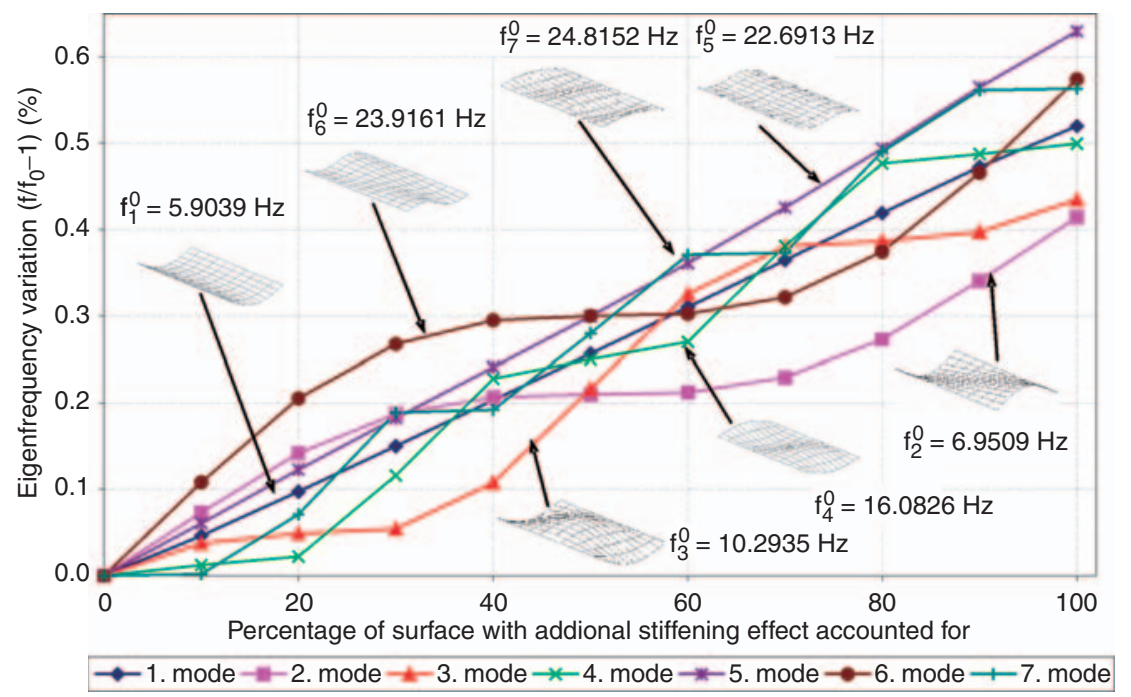

Figure 4. Eigenfrequency variation due to the additional stiffening effect. 
unchanged during the action of the loads. On the other hand, if those assumptions are not met, it means that the structural parameters, such as mechanical stiffness for example, change during the action of loads. Such a behavior is denoted as geometrically non-linear and it requires a geometrically non-linear formulation. It is a matter of engineering judgment whether to perform one or another type of analysis, but the choice needs to be justified. The here considered active structures with embedded/bonded piezoelements belong to the group of slender structures. They are susceptible to relatively large displacements and/or rotations, typically as a consequence of the act of external transverse forces or bending moments, still exhibiting small strains. Displacements in the range of structure's thickness may already suffice to make the geometrically nonlinear analysis necessary in order to achieve required accuracy. Such a magnitude of displacement is not so rare in practical applications of thin-walled piezostructures. It should be emphasized that retaining the small strain range is especially important for the piezoelectric patches, which are most frequently made of rather brittle ceramics.

The adopted geometrically non-linear formulation of the element belongs to the updated Lagrangian formulation (the reference configuration is always the last calculated one) with the corotational formulation taken advantage of in order to approximate natural strains in the local, corotational c.s. An advantage of this approach is that it is well adapted to the treatment of $\mathrm{FE}$ with rotational degrees of freedom (beams, plates, shells) for arbitrarily large rotations. Such elements are difficult to treat with the total Lagrangian formulation due to a complicated update of the rotational degrees of freedom and the requirement to reduce the material law according to the assumption of zero normal stress (or strain) in the thickness direction. An interested reader is referred to Marinković (2007) and Marinković et al. (2008) for more information on the developed formulation.

An incremental step-by-step approach represents a usual solution strategy in the non-linear analysis. Assuming that the solution at discrete time $t-\Delta t$ is known, it seeks the solution at discrete time t, with a suitably chosen time increment $\Delta \mathrm{t}$. The linearized set of FE equations for the piezoelectric continuum can be given in the following form on the element level:

$$
\begin{aligned}
& { }^{\mathrm{t}}[\mathrm{M}]^{\mathrm{t}}\left\{\ddot{\mathrm{u}}_{\mathrm{e}}\right\}+{ }^{\mathrm{t}}[\mathrm{C}]^{\mathrm{t}}\left\{\dot{\mathrm{u}}_{\mathrm{e}}\right\}+{ }^{\mathrm{t}-\Delta \mathrm{t}}\left[\mathrm{K}_{\mathrm{uu}_{\mathrm{T}}}\right]^{\mathrm{t}-\Delta \mathrm{t}}\left\{\Delta \mathrm{u}_{\mathrm{e}}\right\}+{ }^{\mathrm{t}-\Delta \mathrm{t}}\left[\mathrm{K}_{\mathrm{u} \phi}\right]^{\mathrm{t}-\Delta \mathrm{t}} \\
& \left\{\Delta \Phi_{\mathrm{e}}\right\}=\left\{\mathrm{f}_{\mathrm{ext}_{\mathrm{e}}}\right\}-{ }^{\mathrm{t}-\Delta \mathrm{t}}\left\{\mathrm{f}_{\text {inte } \left._{\mathrm{e}}\right\}}\right. \\
& { }^{\mathrm{t}-\Delta \mathrm{t}}\left[\mathrm{K}_{\phi \mathrm{u}}\right]^{\mathrm{t}-\Delta \mathrm{t}}\left\{\Delta \mathrm{u}_{\mathrm{e}}\right\}+{ }^{\mathrm{t}-\Delta \mathrm{t}}\left[\mathrm{K}_{\phi \phi}\right]^{\mathrm{t}-\Delta \mathrm{t}}\left\{\Delta \Phi_{\mathrm{e}}\right\} \\
& ={ }^{\mathrm{t}}\left\{\mathrm{q}_{\mathrm{exte}}\right\}-{ }^{\mathrm{t}-\Delta \mathrm{t}}\left\{\mathrm{q}_{\text {inte }}\right\},
\end{aligned}
$$

where $\left\{\mathrm{f}_{\mathrm{ext}}\right\},\left\{\mathrm{f}_{\mathrm{int}}\right\},\left\{\mathrm{q}_{\mathrm{ext}}\right\}$, and $\left\{\mathrm{q}_{\mathrm{int}}\right\}$ stand for the external and internal mechanical and electrical loads,
$[\mathrm{M}]$ is the mass matrix, $[\mathrm{C}]$ the damping matrix (usually defined as Rayleigh damping), $\left[\mathrm{K}_{\mathrm{uuT}}\right]$, $\left[\mathrm{K}_{\mathrm{u} \phi}\right]$, and $\left[\mathrm{K}_{\phi \phi}\right]$ are the tangential mechanical stiffness, piezoelectric coupling, and dielectric stiffness matrices and the vectors $\left\{\mathrm{u}_{\mathrm{e}}\right\}$ and $\left\{\Phi_{\mathrm{e}}\right\}$ comprise all the element mechanical and electrical degrees of freedom, respectively. The left superscript denotes at which time instant the corresponding quantity is defined, while $\Delta$ denotes the increment of the corresponding quantity between the actual and the next time instant (i.e., between time instants $\mathrm{t}-\Delta \mathrm{t}$ and $\mathrm{t}$ ). The external mechanical and electric loads are the applied forces/moments and electric charges, while the internal mechanical and electrical loads are obtained integrating the mechanical stresses and dielectric displacements over the current structure configuration (i.e., at time t), respectively:

$$
\begin{aligned}
\left\{\mathrm{f}_{\text {int }_{\mathrm{e}}}\right\} & =\int_{\mathrm{V}}\left[\mathrm{B}_{\mathrm{L}}\right]^{\mathrm{T}}\{\sigma\} \mathrm{dV}, \\
\left\{\mathrm{q}_{\text {int }_{\mathrm{e}}}\right\} & =\int_{\mathrm{A}}\left[\mathrm{B}_{\Phi}\right]^{\mathrm{T}}\{\mathrm{D}\} \mathrm{dA},
\end{aligned}
$$

with the integration in (16) running over the volume of the whole structure, $\mathrm{V}$, and in (17) over the surface of the piezopatches, $A,\left[\mathrm{~B}_{\mathrm{L}}\right]$ is the linear straindisplacement matrix and $\left[\mathrm{B}_{\Phi}\right]$ is the electric field electric potential matrix.

If a static case is considered, the dynamic effects, i.e., the inertia and damping, are excluded from (14) and time obviously represents only a convenient auxiliary variable used to denote gradually increasing loads. When it is dealt with a dynamic case, it is convenient to move the terms containing mechanical stiffness and piezoelectric coupling matrices from the left-hand side to the right-hand side of the equation. Adding those terms to the internal force at time instant $t-\Delta t$ gives the internal force at time instant $t,{ }^{t}\left\{f_{\text {inte }_{\mathrm{e}}}\right\}$. Actually, this term is usually calculated using directly the information about the stress state at t, i.e., (16) is used, but the previous observation points out an interesting fact about the piezoelectrically induced loads. Namely, they are configuration dependent, i.e., they are of the follower type. This is due to the fact that the piezoelectrically induced loads change their orientation during deformation as the active elements change their orientation (reflected in the change of the piezoelectric coupling matrix). The intensity of the induced loads is also affected, but to a smaller extent. The same is pretty much valid for the measured voltages, when active elements are used as sensors. As Bathe (1982) has emphasized, the virtual work of the follower forces can be approximated to a sufficient accuracy using a small enough load/time step. Otherwise, the virtual work due to the change of the piezoelectrically induced loads as a consequence of the change in geometry would have to be taken into account, which is a difficult task since both 
the direction and the intensity of the loads change over the incremental step.

The developed element is implemented in an explicit dynamic solver. Such time-marching-forward schemes are performed without iterations and the associated factorization of the structure matrices, but they are only conditionally stable requiring that the time-step is smaller than a critical value. A small time step complies well with the aforementioned follower nature of the induced forces. It also permits to apply small strain small displacement (or engineering) relationship with respect to the co-rotational c.s. in order to calculate the incremental strains. As proposed in Ansys Theory Reference, the strain-displacement matrix of the midconfiguration between two consecutive configurations is used for the purpose. Static cases are solved using the same solver in combination with the dynamic relaxation method. The idea consists in observing the solution of a static problem as a steady-state solution of a dynamic problem. Hence, the static problem is transformed into a dynamic one by adding artificial dynamic effects and parameters so that the degree of freedom of the model with the highest eigenfrequency is critically damped. This actually means that in the dynamic relaxation method both the mass and damping matrices lose their physical background and become fictive quantities used to control the solution process.

In the following, a simple active structure is considered in order to assess the importance of the geometrically non-linear effects in its static and transient behavior.

\section{Static Example}

The initial geometry of the considered structure corresponds to a plate simply supported over two parallel edges (Figure 5). It consists of three layers forming a symmetric architecture. The mid-layer is made of graphite fiber-reinforced epoxy T300/976, with the Young's modulus in the fiber direction $\mathrm{Y}_{11}=150 \mathrm{GPa}$ and in a perpendicular (in-plane) direction $\mathrm{Y}_{22}=9 \mathrm{GPa}$, Poisson's ratio $v_{12}=0.3$ and shear moduli $\mathrm{G}_{12}=7.1 \mathrm{GPa}$ and $\mathrm{G}_{23}=2.5 \mathrm{GPa}$. The orientation of fibers is $90^{\circ}$ with respect to the global $x$-axis and the mass density of the layer is $\rho=3.95 \mathrm{~g} / \mathrm{cm}^{3}$.

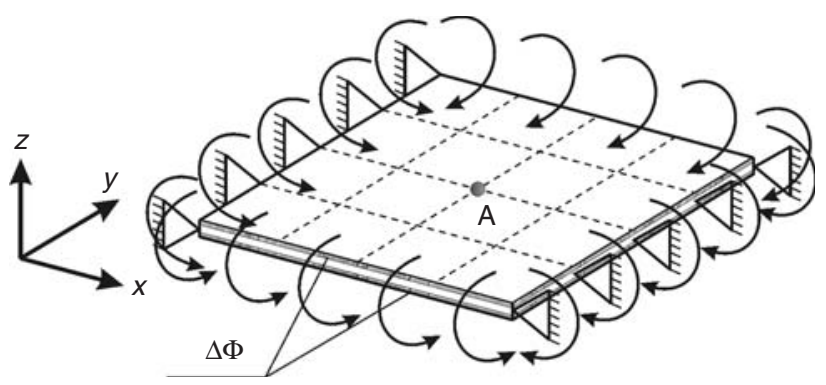

Figure 5. Simply supported composite shell.
The outer two layers are made of piezoelectric ceramic PZT G1195 which is considered to have isotropic mechanical properties: $\mathrm{Y}_{11}=63 \mathrm{GPa}$ and $v_{12}=0.3$. Furthermore, the piezolayers have the in-plane piezoelectric constant $\mathrm{e}_{31}=\mathrm{e}_{32}=22.86 \times 10^{-5} \mathrm{C} / \mathrm{m}^{2}$ and the mass density $\rho=7.85 \mathrm{~g} / \mathrm{cm}^{3}$. The piezolayers are oppositely polarized and when the same electric voltage is supplied to both of them simultaneously a bending moment uniformly distributed over the edges is induced (Figure 5). The point A, which is located in the middle of the structure, is chosen as a representative one and its motion is observed as the deformation of the structures progresses.

The obtained results in the static case are compared with those from the Shell91 element from the ANSYS FE library (Ansys Theory Reference). It is an 8-node layerwise shell element, which applies the $2 \times 2$ in-plane integration rule, but a layerwise numerical integration in the thickness direction (with the ACShell9 the integration in the thickness direction is analytical). It should also be emphasized that the Shell91 is a purely mechanical element, thus not capable of modeling piezoelectric coupling. Thus, this case can not be calculated directly by the Shell91 element and an approximate approach is required. Namely, the moments induced through the piezoelectric coupling are pre-calculated by applying the overall voltage of $300 \mathrm{~V}$ on the initial structure configuration. The socalculated bending moments are then incrementally applied. Hence, in this calculation the follower nature of the induced bending moments is not accounted for because their magnitude and direction are calculated for the initial configuration. Both, the pure (prescribed voltages) and the approximate (pre-calculated moments) approach calculated with the ACShell9 yielded a very small difference in the order of $0.1 \%$. Thus, the conclusion states that the influence of the follower nature of the piezoelectrically induced loads does not play an important role in the case of the considered structure. This means that the results from ANSYS obtained with the pre-calculated bending moments can be used for the purpose of comparison.

Figure 6 shows a high agreement in the results obtained with the ACShell9 and Shell91 elements. It also reveals significant non-linear effects, since the geometrically non-linear result starts to diverge from the linear one already for the voltage of approximately $30 \mathrm{~V}$. At the full voltage of $300 \mathrm{~V}$ the result based on the assumption of linearity overestimates the non-linear result by $89.2 \%$. Such a difference is certainly worth of attention. In the linear analysis of the considered case, bending stiffness plays the major role in the structural behavior. However, due to the selected boundary conditions and the change in the structural configuration, in the actual behavior of the structure significant internal membrane forces are induced very fast after the 
deformation is initiated. Hence, the structure resists the deformation more efficiently than predicted by the linear analysis. Those effects and the corresponding behavior of the structure are successfully predicted by the geometrically non-linear analysis.

\section{Transient Examples}

The transient behavior of the same structure is observed considering input voltage as a harmonic and impulse time functions. It should be emphasized that damping is neglected in both examples, i.e., the damping coefficients are set equal to zero.

In the first case, the electric voltage (excitation) is defined in a sine form with the amplitude of $300 \mathrm{~V}$ and frequency of $100 \mathrm{~Hz}$. The behavior of the structure is observed in a time period of $0.05 \mathrm{~s}$. The linear solution is obtained by the Newmark method $\left(\Delta t=10^{-4} \mathrm{~s}\right.$, i.e., $0.5 \times 10^{3}$ steps) with the Semi-Loof shell element (dashed line in Figure 7) and the ACShell9 element (the two results are seen as almost congruent in the diagram) and by the central difference method $\left(\Delta \mathrm{t}=2 \times 10^{-7} \mathrm{~s}\right.$, i.e., $2.5 \times 10^{5}$ steps) with the ACShell9 element. The nonlinear solution is obtained only by the central difference method $\left(\Delta \mathrm{t}=1 \times 10^{-7}\right.$ s, i.e., $5 \times 10^{5}$ steps $)$ with the

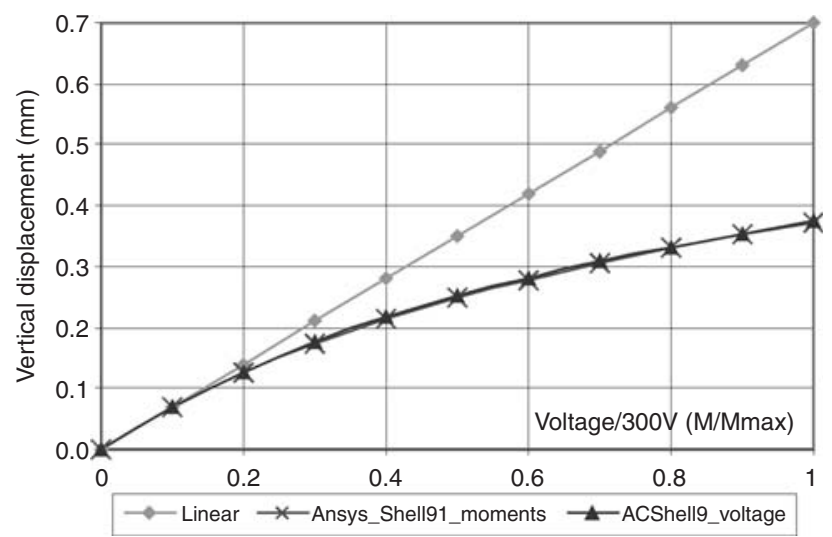

Figure 6. Mid-point vertical displacement.

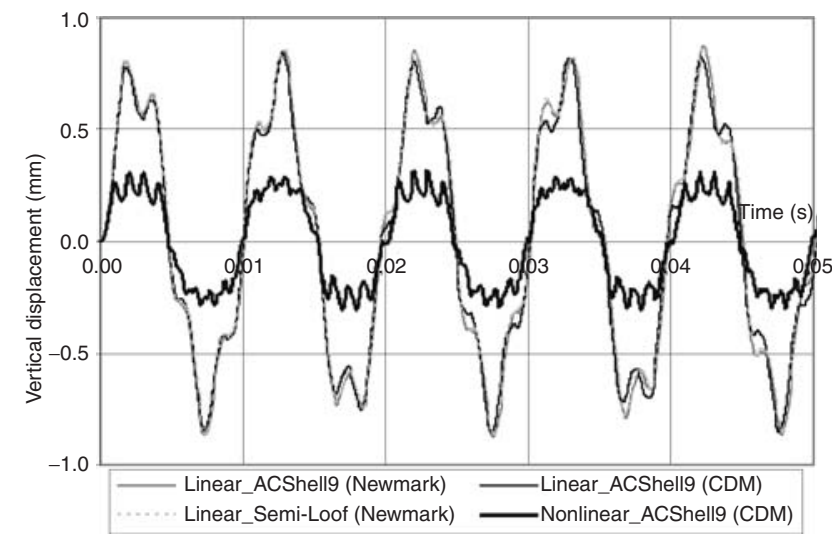

Figure 7. Response to harmonic piezoelectric excitation.
ACShell9 element. The Semi-Loof element is based on the discrete Kirchhoff theory.

It may be noted in Figure 7 that both the linear and non-linear prediction of the dynamic behavior of the structure yield oscillation driven at the frequency of excitation and there is also a structural response in higher frequencies. The amplitudes of the non-linear solution are lower and the response in higher harmonics is obviously shifted to higher frequencies with respect to the response of the linear solution, especially in the vicinity of maximal deflections. Both effects are a consequence of the fact that the structure becomes stiffer as the deformation progresses from the initial configuration to the configuration corresponding to the maximal displacements.

In the second case, the structure is excited by electric voltage in the form of an impulse, the duration of which is $3 \times 10^{-4} \mathrm{~s}$. The amplitude of the voltage is $300 \mathrm{~V}$. The differences in the linear and non-linear structural response are obvious in Figure 8 and the remarks from the previous case extend to this one as well. However, an additional remark may be given considering the two cases in parallel. In the first example a forced oscillation is caused by a harmonic load (electric voltage). The harmonic nature of the load is reflected in both the linear and non-linear prediction of the structural response yielding a certain similarity in the form of the response. In the second case, however, the load is in the form of a short impulse and no such a similarity can be observed any more. Namely, considering the linear and non-linear predictions of the deflection in the second case, it can be noticed that they can even yield relatively large deflections of different signs (opposite orientation of displacements along the $z$-axis) at the very same time instants. For example, the predicted deflection shortly before the time instant $0.001 \mathrm{~s}$ (Figure 8) can be observed to notice this effect. This demonstrates once again that the differences between the linear and geometrically nonlinear prediction can be significant, which talks in favor of a need for the geometrically non-linear formulation.

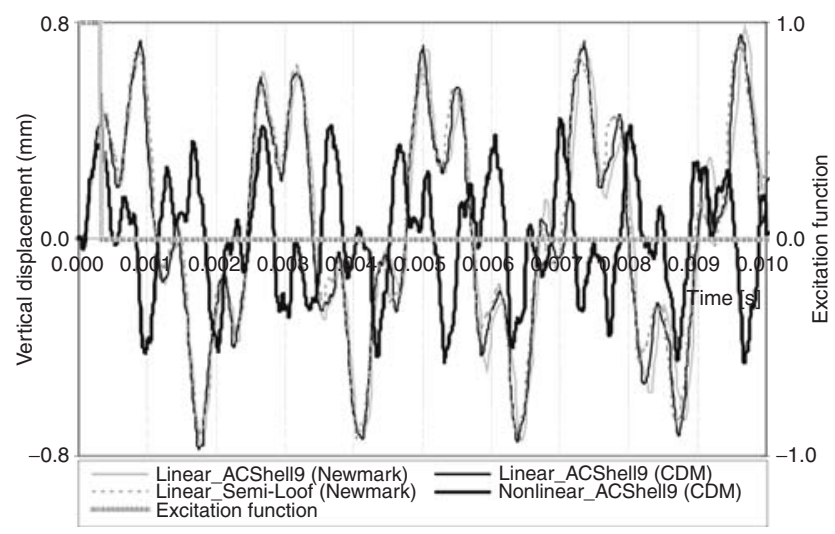

Figure 8. Response to impulse piezoelectric excitation. 


\section{CONCLUSIONS}

The thin-walled piezoelectric active structures have attracted a great deal of attention in the last years. Many researchers have devoted their work to their modeling and implementation in a wide range of products. The intention of the authors was to give here some observations about the modeling of considered structures, which stemmed out from their work in the field.

Regarding modeling of electric field quantities, it is emphasized that their consistent description is closely related to the chosen 2D theory (kinematical assumptions) for modeling thin-walled structures. The sodeveloped functions lead to an additional stiffening effect and the parameters influencing its significance are given. The significance of the effect is considered on a small set of static examples and an example dealing with modal analysis of a plate. All the considered examples clearly demonstrate that for typical application of small, thin piezoelectric patches on a relatively large surface of a host structure, the typical approximations for the electric field and electric potential (given in (4)) yield quite satisfactory results. The applications which would require the use of higher order functions are of rather academic nature. They involve cases where most of the structure's surface is covered by active elements, the thickness of active elements is relatively large compared to the overall thickness of the structure and, additionally, the passive material of the host structure has relatively small mechanical stiffness.

The examples dealing with non-linearities are considered on a relatively simple structure. Nevertheless, both static and transient examples clearly demonstrate that significant non-linear effects may occur shortly after the deformation is initiated, which further on considerably affects the overall behavior of the structure. The nonlinearities are especially pronounced in the cases where the change in the structure configuration in combination with boundary conditions give rise to change in the way the structure essentially resists excitations. Typical cases are problems which are in linear analysis described as bending dominated, but which can pretty fast involve a considerable membrane behavior throughout the deformation of the structure. This was demonstrated in the considered cases. Regarding the coupled-field cases, such as electro-mechanical, there is another issue to be noted. Namely, the piezoelectric actuator forces are of the follower type. They are related to the piezoelectric stiffness matrix which is always integrated with respect to the actual configuration, thus depending on it. Nevertheless, a small enough load (or time) step, allows the virtual work of the follower forces to be approximated to a sufficient accuracy. In the considered cases no significant consequences originating from this issue are recognized, which should not lead to a general conclusion in the same direction.

\section{ACKNOWLEDGMENTS}

This research was partially supported by the German state Saxony-Anhalt and by the Ministry of Science and Technological Development of Republic of Serbia, Project No. ON144002. The authors gratefully acknowledge this support.

\section{REFERENCES}

Ansys Theory Reference. Available at: http://wwwl.ansys.com/ customer/content/documentation/90/ansys/a_thry90.pdf (accessed on 15th February, 2008)

Bathe, K.J. 1982. Finite Element Procedures in Engineering Analysis, Prentice-Hall, Inc., Englewood Cliffs, New Jersey.

Butz, A. and Klinkel, S. 2005. "A Finite Element Formulation for the Non-linear Analysis of Piezoelectric Three-dimensional Beam Structures," PAMM, 5(1):383-384.

Cotoni, V. 2006. "A Finite Element for Piezoelectric Multilayered Plates: Combined Higher-order and Piecewise Linear $\mathrm{C}^{0}$ Formulation," Journal of Intelligent Material Systems and Structures, 17(2):155-166.

Gabbert, U., Görnandt, A. and Köppe, H. 1998. "Benchmark Problems for the Analysis of Piezothermoelastic Smart Structures," In: NAFEMS Seminar: Finite Element Applications for Adaptive Structural Elements, Magdeburg.

Ikeda, T. 1996. Fundamentals of Piezoelectricity, Oxford University Press Inc., New York.

Kulkarni, S.A. and Bajoria, K.M. 2007. "Large Deformation Analysis of Piezolaminated Smart Structures Using Higher-order Shear Deformation Theory," Smart Materials and Structures, 16:1506-1516.

Marinković, D. 2007. "A New Finite Composite Shell Element for Piezoelectric Active Structures," Ph.D. Thesis, Ottovon-Guericke Universität Magdeburg, Fortschritt-Berichte VDI, Reihe 20: Rechnerunterstützte Verfahren, Nr. 406, Düsseldorf.

Marinković, D, Köppe, H. and Gabbert, U. 2006. "Numerically Efficient Finite Element Formulation for Modeling Active Composite Laminates," Mechanics of Advanced Materials and Structures, 13(5):379-392.

Marinković, D., Köppe, H. and Gabbert, U. 2007. "Accurate Modeling of the Electric Field Within Piezoelectric Layers for Active Composite Structures," Journal of Intelligent Material Systems and Structures, 18(5):503-513.

Marinković, D., Köppe, H. and Gabbert, U. 2008. “Degenerated Shell Element for Geometrically Non-linear Analysis of Thin-walled Piezoelectric Active Structures", Smart Materials and Structures, 17:015030.

Piefort, V. 2001. "Finite Element Modeling of Piezoelectric Active Structures," Ph.D. Thesis, Université Libre de Bruxelles.

Rabinovitch, O. 2005. "Geometrically Non-linear Behavior of Piezoelectric Laminated Plates," Smart Materials and Structures, 14:785-798.

Yang, F., Lammering, R. and Mesecke-Rischmann, S. 2004. "Advanced Shell Element Formulations for Coupled Electromechanical Systems," PAMM, 4(1):386-387. 